\title{
SYNTHESIS AND SURFACE CHEMISTRY OF ZN3P2
}

\author{
Gregory M. Kimball, Nathan S. Lewis, Harry A. Atwater \\ California Institute of Technology, Pasadena, CA, USA
}

\begin{abstract}
Zinc phosphide $\left(\mathrm{Zn}_{3} \mathrm{P}_{2}\right)$ is a promising alternative to traditional materials (e.g. CIGS, CdTe, a-Si) for thin film photovoltaics. Open circuit voltage in $\mathrm{Zn}_{3} \mathrm{P}_{2}$ cells has been limited by Fermi-level pinning due to surfaces states and heterojunction interdiffusion, motivating the need to prepare interfaces that are electrically passive and chemically inert. We investigated the surface chemistry of $\mathrm{Zn}_{3} \mathrm{P}_{2}$ via etching with bromine in methanol and passivation with ammonium sulfide in t-butanol. The treatment decreases surface oxidation as determined by $x$ ray photoelectron spectroscopy and provides a stable, low-defect interface as monitored by steady-state photoluminescence. Magnesium Schottky diodes fabricated with sulfur-passivated interfaces show evidence of enhanced barrier heights in comparison to control devices.
\end{abstract}

\section{INTRODUCTION}

$\mathrm{Zn}_{3} \mathrm{P}_{2}$ is an earth-abundant material that has shown promise for solar energy conversion but has not been investigated as thoroughly as other thin film materials. It is one of the very few materials that happens to possess both a direct energy gap near the terrestrial optimum (1.5 $\mathrm{eV}$ ) [1] and earth abundant constituent elements. Predominantly p-type doping has been observed in $\mathrm{Zn}_{3} \mathrm{P}_{2}$, requiring photovoltaic devices to use Schottky barriers and semiconductor heterojunctions. The record solar energy conversion efficiency for a device with a $\mathrm{Zn}_{3} \mathrm{P}_{2}$ absorber was set in 1979, reaching $6.08 \%$ with a diffused $\mathrm{Mg}_{3} \mathrm{P}_{2}$ heterojunction [2]. Despite considerable effort, other device structures using $p-n$ heterojunctions have not demonstrated good performance [3].

The lack of a systematic study of $Z n_{3} P_{2}$ surface and interface chemistry has impeded progress in realizing its potential for solar energy conversion. In particular, the nature of the surface prepared using the traditional etchant, bromine in methanol, has not been fully characterized. Kato et al. used X-ray photoelectron spectra (XPS) to show that an additional phosphorus species remains on the surface, but this species has yet to be conclusively identified [14]. Native oxides grew quickly upon exposure to air, limiting the quality of the surface. Sulfur passivation has been used with $\mathrm{InP}$ and $\mathrm{GaAs}$ materials to decrease surface recombination sites and protect the surface against oxidation in air [16]. Although
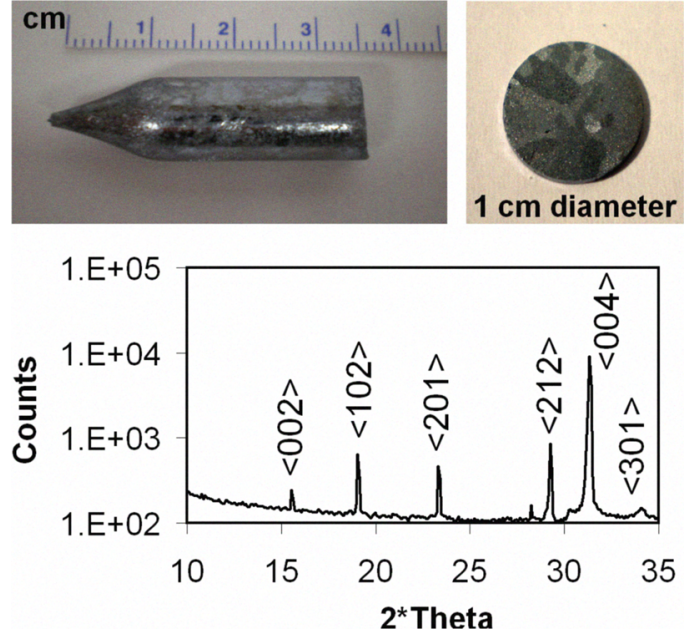

Fig. 1. Photographs of (a) $\mathrm{Zn}_{3} \mathrm{P}_{2}$ boule, (b) unpolished polycrystalline wafer. (c) XRD pattern of polished wafer.

sulfur passivation has been shown to be effective for a number of materials, this study is the first attempt to extend the treatment to $Z n_{3} P_{2}$ surfaces.

\section{EXPERIMENTAL}

\section{Production of $\mathrm{Zn}_{3} \mathrm{P}_{2}$ Wafers}

As $\mathrm{Zn}_{3} \mathrm{P}_{2}$ wafers cannot be purchased commercially, the samples used in this study were synthesized from elemental red phosphorus and zinc metal by established methods [4]. Red phosphorus lump and zinc shot of $99.9999 \%$ purity (Alfa Aesar) were loaded in opposite ends of a quartz tube so that the red phosphorus could be converted to white phosphorus in situ at $500^{\circ} \mathrm{C}$. Then the zinc metal was heated to $850^{\circ} \mathrm{C}$ to allow the vapor phase reaction of white phosphorus and zinc to form raw $\mathrm{Zn}_{3} \mathrm{P}_{2}$ crystals. These crystals were ground into a powder and purified by sublimation in a fresh quartz tube to remove less volatile impurities.

The purified powder was then grown into large $\mathrm{mm}$ grain size boules by physical vapor transport in carboncoated Bridgman quartz ampoules (see Fig. 1a). Approximately $10 \mathrm{~g}$ of $Z \mathrm{n}_{3} \mathrm{P}_{2}$ were loaded with $10 \mathrm{mg}$ of $\mathrm{Ag}$ shot $99.9999 \%$ (ESPI) for in situ doping. Then the ampoule was translated at a rate of 1-2 $\mathrm{mm}$ per day for several weeks into the cold zone of the furnace held at 
$850^{\circ} \mathrm{C}$ [5]. As-grown boules with $\sim 1 \mathrm{~cm}^{2}$ grain size were cylindrical with $1 \mathrm{~cm}$ diameter and about $6 \mathrm{~cm}$ in length.

$\mathrm{Zn}_{3} \mathrm{P}_{2}$ boules were sliced and polished to produce wafers with a mirror finish. An ISOMET low speed diamond saw sliced wafers of approximately $600 \mu \mathrm{m}$ in thickness (see Fig. 1b). These wafers were then polished with a MINIMET polishing instrument using 15, 6, 3, 1, and finally $0.25 \mu \mathrm{m}$ diamond paste. A Philips X'Pert Pro X-ray diffractometer using copper $\mathrm{K}$ - $\alpha \mathrm{X}$-rays was used to collect diffraction patterns that show the wafers to be highly crystalline phase-pure $\mathrm{Zn}_{3} \mathrm{P}_{2}$ (see Fig. 1c) [6].

As-grown wafers have a resistivity of $10^{4}$ to $10^{6} \Omega-\mathrm{cm}$ that can be reduced to $\sim 10^{3} \Omega-\mathrm{cm}$ by annealing in white phosphorus at $400^{\circ} \mathrm{C}$ for 24 hours [7]. Resistivity measurements allowed for an estimate of carrier concentration by the following relation [8]:

$$
\rho=\frac{1}{q\left(n \mu_{n}+p \mu_{p}\right)}
$$

where $n$ is the electron concentration, $\mu_{n}$ is the electron mobility, $p$ is the hole concentration, and $\mu_{p}$ is the hole mobility. Minority carrier conduction was neglected and the reported hole mobility of $20 \mathrm{~cm}^{2} N$ s for $\mathrm{Zn}_{3} \mathrm{P}_{2}$ [4] was used. Wafers used in this study are near intrinsic with an estimated acceptor concentration of $10^{13}$ to $10^{14} \mathrm{~cm}^{-3}$.

\section{Surface Chemistry and Passivation}

Native oxides were removed by etching samples with a $1-3 \%$ by volume $\mathrm{Br}_{2}$ in methanol for one minute and then thoroughly rinsing with methanol [2]. The sulfur passivation solution consisted of a saturated solution of $\left(\mathrm{NH}_{4}\right)_{2} \mathrm{~S}$ in t-butanol [9]. Sulfur treatments were for one minute followed by a methanol dip for one minute to remove excess sulfur solution.

Chemical surface species were analyzed by $x$-ray photoelectron spectroscopy (XPS) immediately after treatment and after prescribed periods of air aging. XPS data were collected using an M-probe spectrometer (VG Instruments) using monochromatic Al K- $\alpha$ X-rays (1486.6 $\mathrm{eV}$ ) that were incident at $35^{\circ}$ from the sample surface. Surface overlayers of element phosphorus, phosphorus sulfides, and native oxides were fit to a standard overlayer-substrate model $[10,11]$ :

$$
d=\lambda_{o v} \sin (\theta) \ln \left(1+\frac{I_{s}^{\infty}}{I_{o v}^{\infty}} * \frac{I_{o v}}{I_{s}}\right)
$$

where $\lambda_{o v}$ is the electron escape depth through the overlayer in $\mathrm{nm}, \theta$ is the angle of incident $\mathrm{x}$-rays, $I_{s}^{\circ} \%_{o v}{ }^{\circ}$ is the ratio of signal intensities of pure samples, $I_{s}$ is the intensity of the signal from the substrate, and $I_{o v}$ is the intensity of the signal from the overlayer. Electron escape depths were calculated for electrons of $128.3 \mathrm{eV}$ using the following relations [11]:

$$
\begin{gathered}
\lambda_{o v}=538 E^{-2}+0.41(a E)^{\frac{1}{2}} \\
a=\left(\frac{A}{1000 \rho N}\right)^{\frac{1}{3}}
\end{gathered}
$$

where $\lambda_{o v}$ is the electron escape depth through the overlayer in $\mathrm{nm}, E$ is the electron kinetic energy signal of the substrate, $a$ is atomic size in $\mathrm{nm}, A$ is the mean of the atomic masses in $\mathrm{kg} / \mathrm{mol}, \rho$ is density in $\mathrm{g} / \mathrm{cm}^{3}$, and $N$ is Avagadro's number. Escape depths of $128.3 \mathrm{eV}$ electrons from elemental phosphorus, phosphorus sulfides, and zinc phosphate were calculated to be $2.5 \mathrm{~nm}, 2.6 \mathrm{~nm}$, and 2.3 $\mathrm{nm}$ respectively.

The surface quality was monitored using steady-state photoluminescence (PL) intensity. PL spectra were collected with a Princeton Instruments SPEC-10 visible/infrared CCD using excitation from the $488 \mathrm{~nm}$ line of an Argon laser. Spectra were collected before surface treatment and every two minutes thereafter.

\section{Barrier Height Calculations}

Optically thick magnesium films probed the junction properties of $\mathrm{Mg}-\mathrm{Zn}_{3} \mathrm{P}_{2}$ Schottky diodes [2]. $\mathrm{Zn}_{3} \mathrm{P}_{2}$ wafers were etched and then half were subjected to the sulfur treatment previously described. Then they were loaded into a RF plasma sputtering system for the deposition of a $200 \mathrm{~nm} \mathrm{Mg}$ rectifying front contact. As soon as the sputtering was complete, the wafers were immediately loaded into an evaporator for deposition of a $250 \mathrm{~nm} \mathrm{Ag}$ ohmic back contact.

Current-voltage data were collected with a Solarton model 1286 electrochemical analyzer using a scan rate of $20 \mathrm{mV} / \mathrm{sec}$. Values for the diode ideality factor $\eta$ and the reverse bias saturation current $J_{0}$ were derived from fits to the ideal diode equation [8]:

$$
\begin{gathered}
J(V)=J_{0} *\left(\exp \left(\frac{q V}{k T \eta}\right)-1\right) \\
\phi_{B p}=\frac{k T}{q} * \ln \left(\frac{A^{* *} T^{2}}{J_{0}}\right)
\end{gathered}
$$

where $k$ is the Boltzmann constant, $T$ is temperature in Kelvin, $q$ is the charge of the electron, $\Phi_{B p}$ is the barrier height for holes, and $A^{* *}$ is the Richardson's constant (value for Si used [8]).

Capacitance-voltage data was collected using a Schlumberger BI 1260 Impedance/Gain Phase Analyzer across a range of frequencies from $10 \mathrm{~Hz}$ to $100 \mathrm{kHz}$. Frequency dependent traces were collected using a 10 
$\mathrm{mV}$ alternating bias imposed upon a -0.1 to $-2.0 \mathrm{~V}$ constant bias. Barrier heights and carrier concentrations were calculated from the Mott-Schottky equation [8]:

$$
\begin{gathered}
C^{-2}(V)=-A^{2}\left(\frac{2}{q \varepsilon_{s} \varepsilon_{0} N_{A}}\right)\left(V_{b i}+V-\frac{k T}{q}\right) \\
\phi_{B p}=-V_{b i}+\frac{k T}{q}\left(\ln \left(\frac{N_{V}}{N_{A}}\right)+1\right)
\end{gathered}
$$

where $C$ is junction capacitance, $A$ is device area, $\varepsilon_{s} \varepsilon_{0}$ is the dielectric constant of the semiconductor (value for $\ln P$ used [8]), $N_{A}$ is the acceptor density, $V_{b i}$ is the built-in voltage, and $N_{V}$ is the density of states in the valence band (value for InP used [12]).

\section{DATA AND RESULTS}

\section{X-Ray Photoelectron Spectra}

XPS spectra of the P2p and $Z n 2 p^{3 / 2}$ peaks of the aspolished surface showed a stoichiometric surface (3:2 $\mathrm{Zn}: \mathrm{P})$ with substantial phosphorus and zinc oxides. We assign $133.2 \mathrm{eV}$ to $\mathrm{P} 2 \mathrm{p}$ phosphorus oxide, $128.3 \mathrm{eV}$ to $\mathrm{P} 2 \mathrm{p}$ phosphide, and 1022.8 to $\mathrm{Zn} 2 \mathrm{p}^{3 / 2}$ zinc oxide (see Fig. $2 a, b)$ [13]. The underlying $Z_{3} \mathrm{P}_{2}$ was exposed by argon ion sputtering for several minutes, showing clean $P 2 p$ and $\mathrm{Zn} 2 \mathrm{p}^{3 / 2}$ peaks (see Fig. $2 \mathrm{c}, \mathrm{d}$ ).

Upon treatment of the as-polished surface with $1 \% \mathrm{v} / \mathrm{v}$ $\mathrm{Br}_{2}$ in methanol for $30 \mathrm{sec}$, the resulting surface no longer showed sign of oxidation. There was no bromine detectable on the surface within the limits of XPS, but another phosphorus $2 p$ doublet at $130.0 \mathrm{eV}$ was observed distinct from the P2p phosphide peak (see Fig. 3a). Although previous authors have attributed this peak to phosphorus sub-oxides [14], we assign it to elemental phosphorus [15]. The overlayer model (2) predicts that about 2 monolayers of elemental phosphorus remain on the surface. As the etched surface aged, both zinc and phosphorus oxides grew in within the first few hours.

Next, we passivated the $\mathrm{Zn}_{3} \mathrm{P}_{2}$ surface using an ammonium sulfide treatment that has been shown to be effective for stabilizing the surface of III-V semiconductors [16]. Immediately after treatment, the P2p spectrum showed a new species attributable to P-S bonding at $132.1 \mathrm{eV}$ (see Fig. 3c). A nearly 1:1 phosphorus sulfide film was estimated to be about 4 monolayers in thickness. Studies with sulfur passivation of indium phosphide surfaces have assigned S2p signals at $162.1 \mathrm{eV}$ to polysulfide [17], which corresponds well with the signal at $162.3 \mathrm{eV}$ seen in these samples. As the sulfur treated samples aged in air, phosphorus oxides grew in, but the zinc peak did not show strong evidence of oxidation. A shift in the $\mathrm{Zn} 2 \mathrm{p}^{3 / 2}$ peak to $1022.4 \mathrm{eV}$ could be attributed to $\mathrm{Zn}-\mathrm{S}$ bonding, but not conclusively. Additionally, the sulfur S2p signal appears unaffected by the air aging.
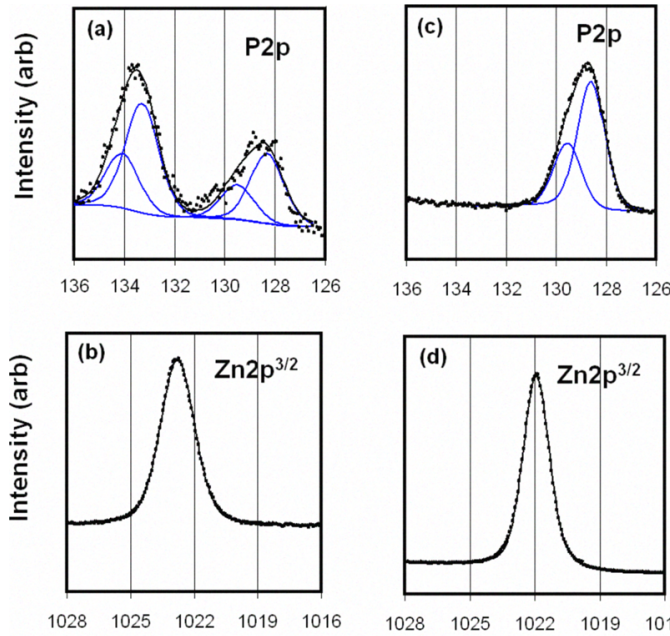

Binding Energy (eV)

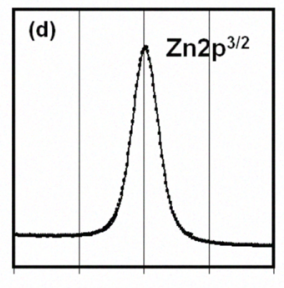

$1028 \quad 1025 \quad 1022 \quad 1019 \quad 1016$

Binding Energy (eV)

Fig. 2. XPS spectra of (a) P2p and (b) $Z n 2 p^{3 / 2}$ for the as polished surface showing native oxide. Spectra of (c) P2p and (d) $Z n 2 p^{3 / 2}$ for argon ion etched surface showing clean $\mathrm{Zn}_{3} \mathrm{P}_{2}$.
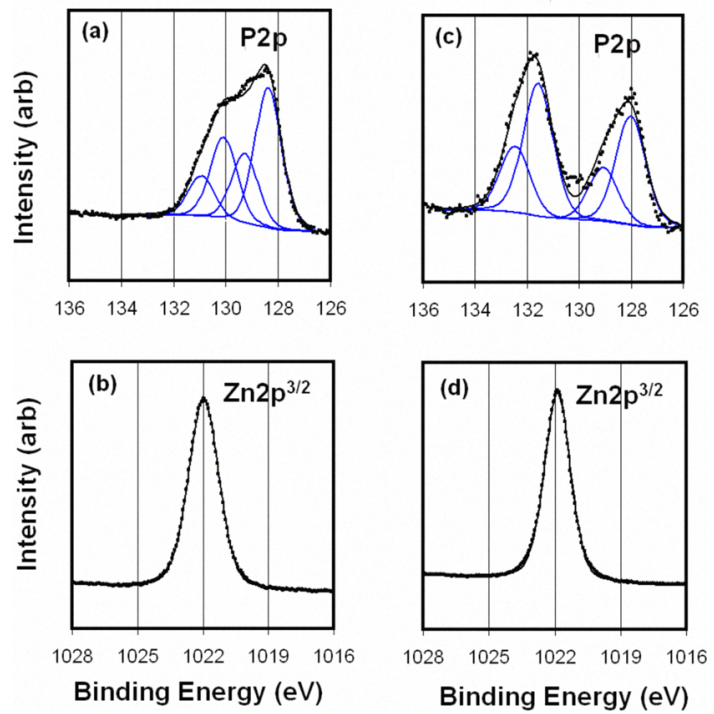

Fig. 3. XPS spectra of (a) P2p and (b) Zn2p $p^{3 / 2}$ for the bromine etched surface showing residual elemental phosphorus. Spectra of (c) P2p and (d) $Z n 2 p^{3 / 2}$ for sulfur treated surface showing phosphorus sulfides.

\section{Steady-State Photoluminescence}

Polished wafers showed a weak signal around 830 $\mathrm{nm}$ near the band edge of $\mathrm{Zn}_{3} \mathrm{P}_{2}$. Upon etching with bromine in methanol, steady-state photoluminescence was greatly enhanced but as the sample oxidized in air this signal lost intensity. After several hours the signal returned to intensities comparable to untreated samples. 

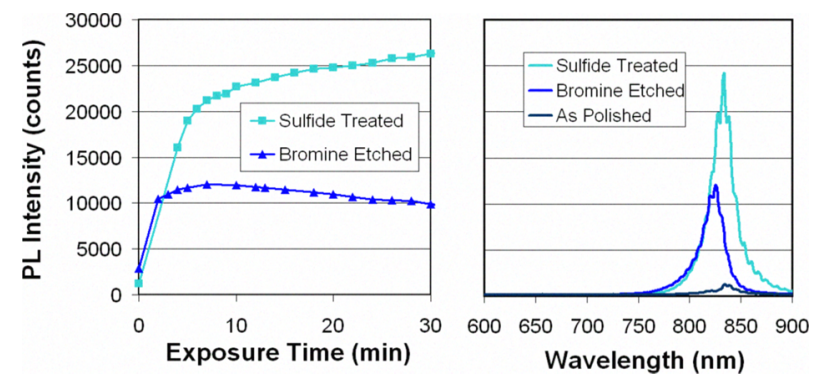

Fig. 4. Steady-state photoluminescence intensity during air exposure at room temperature for samples as polished, freshly etched with $3 \% \mathrm{v} / \mathrm{v} \mathrm{Br}$ in $\mathrm{MeOH}$ for $1 \mathrm{~min}$, and freshly etched and soaked in sat $\left(\mathrm{NH}_{4}\right)_{2} \mathrm{~S}$ in $\mathrm{t}$-ButOH for $1 \mathrm{~min}$.

Sulfide passivated wafers show larger emission intensity compared to etched wafers (see Fig. 4). Even more striking, they exhibited a rise in photoluminescence intensity over time. The increasing steady-state room temperature photoluminescence intensity continues for the first few hours, but gradually fell to the intensity of the aspolished wafers over a period of 24 hours.

\section{Dark Current-Voltage Characteristics}

Dark current-voltage measurements revealed strongly rectifying behavior in the magnesium Schottky diodes (see Fig. 5). Samples displayed a small leakage current that grew exponentially with reverse bias. In forward bias, the devices adhere to the ideal diode equation well for several hundred millivolts before large series resistance dominates the behavior. The bulk resistivity of the wafers currently limits the performance of the diodes for photovoltaic applications. Fits to the ideal diode equation $(5,6)$ using the first few hundred millivolts of forward bias show striking differences between devices fabricated with and without sulfur passivation (see Table 1). Diode ideality factors of $1.55+/-0.08$ were found for samples treated with sulfur, while ideality factors of $2.40+/-0.15$ were found for samples prepared without sulfur passivation. Barrier heights estimated from $J_{0}$ showed an improvement for samples treated with sulfur, increasing from $844+/-12 \mathrm{mV}$ to $867+/-6 \mathrm{mV}$. These results compare favorably with literature reported barrier heights of $800 \mathrm{mV}$ [2].

\section{Capacitance-Voltage Characteristics}

Frequency-dependent impedance measurements showed evidence for even greater barrier heights. The sulfur-passivated devices following the Mott-Schottky equation $(7,8)$ gave barrier heights as high as $983 \mathrm{mV}$ (see Fig. 6). Although some devices performed ideally across a range of frequencies, many of the devices tested had significant frequency dispersion in the derived barrier heights. Carrier concentrations calculated from the slopes of $1 / C^{2}-V$ traces corresponded well to estimates from fourpoint resistivity measurements (see Table 2). Preliminary results suggested that sulfur passivation enhanced barrier
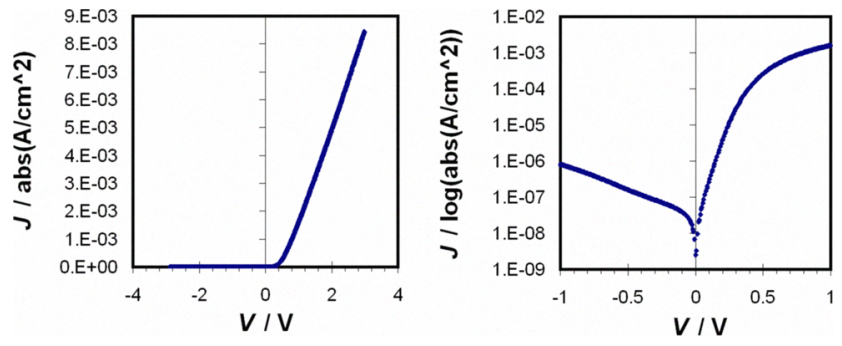

Fig. 5. Dark J-V traces of $Z n_{3} P_{2}-M g$ Schottky diodes.

\begin{tabular}{|c|c|c|c|}
\hline Sulfur & $\boldsymbol{n}$ & $\mathbf{J}_{\mathbf{0}}\left(\mathbf{A} \mathbf{c m}^{\mathbf{2}}\right)$ & $\boldsymbol{\Phi}_{\mathbf{E P}}{ }^{\mathbf{J - V}} \mathbf{( V )}$ \\
\hline Yes & 1.48 & $2.66 \mathrm{E}-08$ & 0.870 \\
Yes & 1.64 & $3.77 \mathrm{E}-08$ & 0.861 \\
Yes & 1.54 & $2.53 \mathrm{E}-08$ & 0.872 \\
\hline No & 2.52 & $1.02 \mathrm{E}-07$ & 0.836 \\
No & 2.23 & $9.45 \mathrm{E}-08$ & 0.838 \\
No & 2.45 & $4.32 \mathrm{E}-08$ & 0.858 \\
\hline
\end{tabular}

Table 1. Parameters derived from J-V measurements.

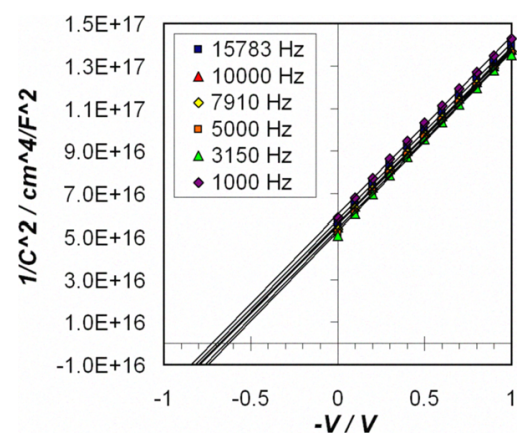

Fig. 6. Capacitance-Voltage traces of $\mathrm{Zn}_{3} \mathrm{P}_{2}-\mathrm{Mg}$ Schottky diodes.

\begin{tabular}{|c|c|c|c|}
\hline Sulfur & $\boldsymbol{\Phi}_{\mathrm{Bp}}{ }^{\mathrm{C}-\mathrm{V}}(\mathbf{V})$ & $\mathbf{N}_{\mathrm{A}}{ }^{\mathrm{C}-\mathrm{V}} \mathbf{( \mathbf { c m } ^ { - 3 } )}$ & $\left.\mathbf{N}_{\mathrm{A}}{ }^{{ }} \mathbf{c m}^{-3}\right)$ \\
\hline Yes & 0.641 & $3.29 \mathrm{E}+13$ & $5.97 \mathrm{E}+13$ \\
Yes & 0.924 & $8.97 \mathrm{E}+13$ & $1.72 \mathrm{E}+13$ \\
Yes & 0.983 & $1.35 \mathrm{E}+14$ & $9.64 \mathrm{E}+13$ \\
\hline
\end{tabular}

Table 2. Parameters derived from capacitance-voltage and resistivity measurements.

heights in $\mathrm{Zn}_{3} \mathrm{P}_{2}-\mathrm{Mg}$ Schottky diodes by almost $200 \mathrm{mV}$ as compared to those reported in the literature [2].

\section{DISCUSSION AND FUTURE WORK}

Nearly all previous work involving $\mathrm{Zn}_{3} \mathrm{P}_{2}$ used a 1-3\% solution by volume of bromine in methanol, but little characterization has been performed of the resulting surface. The mechanism of the etching process is thought to occur as follows [18]: 


$$
\begin{aligned}
& \mathrm{Zn}_{3} \mathrm{P}_{2}+6 \mathrm{Br}_{2} \rightarrow 3 \mathrm{ZnBr}_{2}+2 \mathrm{PBr}_{3} \\
& \mathrm{PBr}_{3}+3 \mathrm{CH}_{3} \mathrm{OH} \rightarrow\left(\mathrm{CH}_{3} \mathrm{O}\right)_{2} \mathrm{POH}+\mathrm{CH}_{3} \mathrm{Br}+2 \mathrm{HBr}
\end{aligned}
$$

Etched samples were consistently phosphorus-rich, suggesting that the etching process was not completely congruent. Even diluted bromine solutions resulted in $\mathrm{Zn}_{3} \mathrm{P}_{2}$ surfaces with a similar residue of elemental phosphorus several monolayers in thickness. The elemental phosphorus resisted rinsing and sonication, motivating future work to explore etchants for its removal. To date, chemical methods have not established a preparation for clean, stoichiometric $\mathrm{Zn}_{3} \mathrm{P}_{2}$ surfaces.

Combined AES and XPS sputter profiling has shown the etched $Z n_{3} P_{2}$ surface to be susceptible to reaction with $\mathrm{Mg}$. Ireland et al. demonstrated that $\mathrm{a} \mathrm{Mg}_{3} \mathrm{P}_{2}$ layer about 20-40 nm in thickness formed at the metal-semiconductor interface [19]. We hypothesize that treatment with ammonium sulfide helps prevent Mg diffusion by forming a buffer layer of amorphous magnesium phosphide-sulfide alloy. The resulting interface has fewer recombination sites, allowing for better diode ideality factors. MgS may act as a barrier for hole injection across the $M g-Z n_{3} P_{2}$ interface, allowing for increased barrier heights. XPS sputter profiling studies will help discern the efficacy of buffer layers at the metal-semiconductor interface.

Low effective carrier concentrations limited the performance of the devices in this report. We have made recent progress in the diffusion doping of $\mathrm{Zn}_{3} \mathrm{P}_{2}$ wafers with $\mathrm{Ag}$. As-grown wafers of $10^{4}$ to $10^{5} \Omega-\mathrm{cm}$ resistivity were etched as previously described, and then $100 \mathrm{~nm}$ of $\mathrm{Ag}$ were evaporated on the front of the wafer. After an annealing treatment of $550^{\circ} \mathrm{C}$ for $24 \mathrm{hrs}$ to drive in the silver, both sides of the wafer had resistivities of about 1$10 \Omega-\mathrm{cm}$. This corresponds to an increase of nearly four orders of magnitude in carrier concentration, raising the wafers to an estimated $10^{17}$ to $10^{18} \mathrm{~cm}^{-3}$ acceptors. Continuing work will explore the effects of various surface treatments on the performance of high-quality $\mathrm{Mg}-\mathrm{Zn}_{3} \mathrm{P}_{2}$ Schottky diodes.

\section{ACKNOWLEDGEMENTS}

This work was supported by the Office of Energy Efficiency and Renewable Energy, US Department of Energy under grant DE-FG36-08G018006. One of us (GMK) acknowledges support under an NDSEG graduate fellowship. We also acknowledge use of facilities supported by the Center for Science and Engineering of Materials, an NSF MRSEC.

\section{REFERENCES}

[1] E.A. Fagen, "Optical-Properties of Zn3P2", J. Appl. Phys. 50, 1979, pp. 6505-6515.

[2] M. Bhushan, A. Catalano, "Polycrystalline Zn3P2 Schottky-Barrier Solar-Cells", Appl. Phys. Lett. 38, 1, 1981, pp. 39-41.
[3] M. Ginting, J.D. Leslie, "Preparation and ElectricalProperties of Heterojunctions of $\mathrm{ZnO}$ on $\mathrm{Zn3P2}$ and CdTe", Can. J. Phys. 67, 4, 1989, pp. 448-455.

[4] F.C. Wang et al, "Single-Crystal Growth of Zn3P2", J. Crys. Growth, 55, 2, 1981, pp. 268-272.

[5] S. Fuke et al., "Growth and Characterization of Zinc Phosphide Crystals", J. Crys. Growth, 87, 4, 1988, pp. 567-570.

[6] M. von Stackelberg et al., Zeitschrift fuer Physikalische Chemie, Abteilung B: Chemie der Elementarprozesse, Aufbau der Materie, 28, 1935, pp. 427-460.

[7] J. Misiewicz, F. Krolicki et al., "Growth of Zn3P2 Crystals By Gas-Transport Method", Acta Phys Polonica A. $69,6,1986$, pp.1127-1130.

[8] S.M. Sze, K.K. Ng, Physics of Semiconductor Devices. 3rd Edition Wiley 2007.

[9] Y. Fukuda, Y. Suzuki et al., "(NH4)2Sx-Treated InP (001) Studied By High-Resolution X-Ray PhotoelectronSpectroscopy", J. Appl. Phys. 76, 5, 1994, pp. 3059-3062.

[10] K.E. Pomykal, A.M. Fajardo, N.S. Lewis, "Stability of $\mathrm{N}-\mathrm{Si} / \mathrm{CH} 3 \mathrm{OH}$ Contacts as a Function of the Reorganization Energy of the Electron-Donor", J. Phys. Chem. 99, 20, 1995,99, pp. 8302-8310.

[11] D. Briggs, M.P. Seah, Practical Surface Analysis 2nd Edition. John Wiley \& Sons: New York 1990.

[12] Shmidt Handbook Series on Semiconductor Parameters, 1, M. Levinshtein, S. Rumyantsev and M. Shur, ed., World Scientific, London, 1996, pp. 169-190.

[13] Y. Kato, S. Kurita, "Native Oxides on Etched Zn3P2 Surfaces Studied by X-Ray Photoelectron Spectroscopy", Appl. Phys. Lett. 52, 25, 1988, pp. 2133-2135.

[14] U. Elrod, M.C. Luxsteiner, M. Obergfell et al., "Surface-Chemistry of Zn3P2 Single Crystals Studied by XPS” Appl. Phys. B, 43, 3, 1987, pp. 197-201.

[15] C.E. Myers, H.F. Franzen, J.W. Anderegg, "X-Ray Photoelectron-Spectra and Bonding in Transition-Metal Phosphides", Inorg. Chem. 24, 12, 1985, pp. 1822-1824.

[16] H. Oigawa, J.F. Fan, et al., "Universal Passivation Effect of (NH4)S2x Treatment on the Surface of III-V Compound Semiconductors", Jap. J. Appl. Phys. 2-Lett. 30, 3A, 1991, pp. L322-L325.

[17] R. lyer et al., "Sulfur as a Surface Passivation for InP", Appl. Phys. Lett. 53, 2, 1988, pp. 134-136. 
[18] K. Strubbe, W. Gomes, "Bromine-Methanol as an Etchant for Semiconductor - A Fundamental-Study on GaP”, J. Electrochem. Soc. 146, 11, 1993, pp. 3294-3300.

[19] L.L. Kazmerski, P.J. Ireland, A. Catalano, "Surface and Interface Properties of Zn3P2 solar cells", J. Vac. Sci. Technol. 18, 2, 1981, pp. 368-371. 